\title{
Vitamin A deficiency in patients with hepatitis C virus-related chronic liver disease
}

\author{
W. A. F. Peres ${ }^{1 *}$, G. V. Chaves $^{1}$, J. C. S. Gonçalves ${ }^{2}$, A. Ramalho ${ }^{3}$ and H. S. M. Coelho ${ }^{4}$ \\ ${ }^{1}$ Department of Nutrition and Dietetics, Institute of Nutrition, Federal University of Rio de Janeiro, 122 Aureliano Pimentel \\ Street, 21931-300, Rio de Janeiro, Brazil \\ ${ }^{2}$ Department of Medicine, Faculty of Pharmacy, Federal University of Rio de Janeiro, Rio de Janeiro, Brazil \\ ${ }^{3}$ Department of Social and Applied Nutrition, Institute of Nutrition, Federal University of Rio de Janeiro, Rio de Janeiro, \\ Brazil \\ ${ }^{4}$ Section of Hepatology, University Hospital of the Federal University of Rio de Janeiro, Rio de Janeiro, Brazil \\ (Received 12 October 2010 - Revised 9 March 2011 - Accepted 21 March 2011 - First published online 8 June 2011)
}

\section{Abstract}

Hepatitis C virus (HCV) infection is associated with oxidative stress and vitamin A possesses antioxidant activity. The objective of the present study was to investigate vitamin A nutritional status in chronic hepatitis, liver cirrhosis and hepatocellular carcinoma (HCC), according to biochemical, functional and dietetic indicators correlating these findings with liver function, liver damage and death. Vitamin A nutritional status was analysed by serum retinol levels, dietetic indicators and functional indicators. A total of 140 patients with HCVrelated liver disease were enrolled. Vitamin A deficiency was detected in $54.3 \%$ of all patients, and there was a progressive drop in serum retinol levels from chronic hepatitis $\mathrm{C}$ patients towards cirrhosis and HCC patients. Increased total bilirubin, liver transaminases and prothrombin time, presence of hepatic encephalopathy and ascites were related to reduced serum retinol levels, and values $\leq 0 \cdot 78 \mu \mathrm{mol} / 1$ of serum retinol were associated with liver-related death. A high prevalence of inadequate intake of vitamin A was observed in all stages of chronic liver disease. The functional indicator was not an adequate parameter for evaluating the vitamin A nutritional status. Therefore, serum retinol concentration is related to severity of the disease, liver complications and mortality. The effectiveness of nutritional counselling and measures of intervention in this group in improving vitamin A nutritional status should be examined further in a controlled study.

Key words: Vitamin A deficiency: Hepatitis C virus infection: Retinol: Liver disease

The hepatitis $\mathrm{C}$ virus (HCV) is a major causative agent of chronic liver disease (CLD), which often leads to chronic hepatitis, cirrhosis and hepatocellular carcinoma (HCC). To date, HCV infection represents the leading cause of liver transplant in Europe and the USA ${ }^{(1,2)}$. HCV infection is associated with oxidative stress, which plays a crucial role in the onset and progression of liver disease and may contribute to the development of HCC in these patients, as oxidative stress activates hepatic stellate cells, resulting in hepatic fibrosis ${ }^{(3,4)}$.

Vitamin A deficiency (VAD) is one of the world's greatest malnutrition problems. According to the World Health Organization $^{(5)}$, Brazil is classified as having a high prevalence of subclinical VAD. Vitamin A and its retinoid derivatives are essential for physiological functions, including vision, cellular proliferation and differentiation and immune system activity. Night blindness (NB) is a disorder frequently caused by VAD and is defined as diminished night vision in individuals with normal daytime vision ${ }^{(6)}$.

Theoretically, low levels of serum retinol and inadequate intake of vitamin A may be a risk factor for the progression of CLD in HCV patients, considering the potent ability of vitamin A to combat free radicals and its role in suppressing tumour promotion and progression ${ }^{(7,8)}$. In fact, findings from another study ${ }^{(9)}$ have previously demonstrated that lower levels of serum retinol may promote hepatocarcinogenesis in cirrhotic patients. In a normal liver, hepatic stellate cells are major storage sites for vitamin A. Following the activation of hepatic stellate cells, a loss of the characteristic stored intracellular vitamin A occurs; however, it is still unclear whether vitamin A loss causes activation, potentiates it or whether it is simply an event in the cascade of changes that occur during activation ${ }^{(10,11)}$.

Abbreviations: ALT, alanine transaminase; AST, aspartate transaminase; CLD, chronic liver disease; HCC, hepatocellular carcinoma; HCV, hepatitis C virus; MAMC, mid-arm muscle circumference; NB, night blindness; TST, triceps skinfold thickness; VAD, vitamin A deficiency.

*Corresponding author: Dr W. A. F. Peres, fax +552122808343, email wilza@nutricao.ufrj.br 
Considering the importance of VAD on CLD progression in HCV patients, the aim of the present study was to evaluate vitamin A nutritional status in all stages of CLD, including chronic hepatitis, liver cirrhosis and HCC superimposed on liver cirrhosis, according to biochemical, functional and dietetic indicators.

\section{Experimental methods}

In the cross-sectional phase of the present study, data were collected from September 2006 to December 2009 at the University Hospital of the Federal University of Rio de Janeiro, Rio de Janeiro, Brazil. The present study was conducted according to the guidelines laid down in the Declaration of Helsinki, and all procedures involving human subjects/patients were approved by the Research Ethics Committee of the University Hospital. Written informed consent was obtained from all subjects/patients.

Patients aged $\geq 18$ years with HCV-related chronic disease (serum HCV-RNA) were eligible for enrolment. The diagnosis of liver cirrhosis was based on clinical manifestations and laboratorial testing, as well as on ultrasonographic imaging and histological evaluation whenever necessary. Diagnosis of HCC was based on a computerised tomography and/or magnetic resonance findings and serum $\alpha$-fetoprotein. The number of liver-related deaths was recorded during the observation period. All patients in the cross-sectional sample were followed for at least 17 months. The criteria for exclusion were disabsorptive syndromes, type 1 diabetes mellitus, respiratory or CVD, cancer except HCC, cholestasis, chronic renal failure, amyloidosis, pregnancy, chronic and acute alcoholism, use of supplements or drugs containing vitamin A during the last 6 months and any other aetiological factor besides HCV infection.

\section{Evaluation of vitamin A nutritional status}

The evaluation of the nutritional status vitamin A of included biochemical indicators, i.e. serum retinol concentration, dietetic indicators and functional indicators, i.e. NB.

\section{Assessment of serum retinol}

For determining serum retinol concentration, $5 \mathrm{ml}$ of fasting blood sample were taken intravenously from patients on a $12 \mathrm{~h}$ fast. Separation and quantification of the serum retinol were carried out by a validated method for online solidphase extraction coupled with HPLC-MS (Sigma Chemical Company, St Louis, MO, USA). All trans-retinol was used as the reference standard for analysis. The serum retinol values obtained were compared with the cut-off points for normalcy proposed by the World Health Organization ${ }^{(12)}$, and thus were presented in $0.35 \mu \mathrm{mol} / 1$ interval classes. In this manner, VAD was classified as severe deficiency $(<0.35 \mu \mathrm{mol} / \mathrm{l})$, moderate deficiency $(\geq 0.35 \mu \mathrm{mol} / 1<0.70 \mu \mathrm{mol} / \mathrm{l})$ and mild deficiency $(\geq 0.70 \mu \mathrm{mol} / 1<1.05 \mu \mathrm{mol} / \mathrm{l})$. In the present study, a value of $\geq 1.05 \mu \mathrm{mol} / 1$ of serum retinol was considered to be adequate, with a cut-off point of $<1.05 \mu \mathrm{mol} / 1$ indicating $\mathrm{VAD}^{(13)}$.

\section{Retinol assays}

Serum retinol concentrations were measured by a validated method for online solid-phase extraction coupled with HPLC-MS. To minimise retinol photoisomerisation, all processing steps were performed in a room with only a low-intensity light source. Solid-phase extraction was performed on a Prospekt $2^{\mathrm{TM}}$ system (Spark Holland, Emmen, The Netherlands). We used an automated low-pressure gradient system from Shimadzu Company (Tokyo, Japan) with LC-10ADvp solvent delivery, DGU-10B degasser, SCL-10Avp system controller and CTO-10Acvp for column temperature control. The separation of individual analytes was achieved using a Shim-pack CLC-C8 $150 \mathrm{~mm} \times 4.6 \mathrm{~mm}, 5 \mu \mathrm{m}$ particles (Shimadzu Company), analytical column preceded by a Supelco 1/16", peek, $2 \mu \mathrm{m}$ frit, pre-column filter (Supelco, Bellefonte, PA, USA). Solid-phase extraction elution and analyte transfer to the HPLC-MS system were performed using a mobile phase, which consisted of the following solvent mixtures: mixture A, water-acetonitrile (50:50, v/v) with $0 \cdot 1 \%$ formic acid and mixture B, acetonitrile-methyl-tert-butyl diethyl ether (90:10, v/v) with $0 \cdot 1 \%$ formic acid. Analyte elution was performed with a gradient: from $65 \%$ of mixture A to $90 \%$ of mixture $\mathrm{B}$ at a flow rate of $1.1 \mathrm{ml} / \mathrm{min}$ in a $2 \mathrm{~min}$ total runtime. In order to obtain MS, we used a triple-stage quadrupole Quattro LC system with atmospheric pressure chemical ionisation interface of Micromass-Waters (Wythenshawe, UK), acquired in full-scan and under selected-ion monitoring acquisition modes. All transretinol characterisation was carried out using $1.0 \mu \mathrm{g} / \mathrm{ml}$ of standard (Sigma-Aldrich) methanol infused individually into the mass spectrometer at a flow rate of $50 \mu \mathrm{l} / \mathrm{min}$. Optimised variables were averaged for the range of retinol concentrations in human serum. A positive ion mode was used. Because retinol fragments during positive ion atmospheric pressure chemical ionisation form a base peak of $m / z$ 269, selected ion monitoring was used to record the signals at $\mathrm{m} / z 269$. Data acquisition, peak integration and calculations were performed using MassLynx software (Waters Corporation, Milford, MA, USA) residing in a Compaq computer.

\section{Assessment of dietary vitamin A intake}

Vitamin A dietary intake was assessed by a validated semiquantitative food intake questionnaire ${ }^{(14)}$ and was analysed using the food composition table from the Institute of Nutrition of Central America and Panama (Guatemala City, Guatemala) based on the biological activity of retinol, $\beta$-carotene and other carotenoids. According to the RDA, a cut-off value $<900 \mathrm{mg} / \mathrm{d}$ for males and $<700 \mathrm{mg} / \mathrm{d}$ for females was used to define low vitamin A intake ${ }^{(15)}$.

\section{Assessment of night blindness}

The functional indicator NB was assessed by standardised interviews proposed by the World Health Organization ${ }^{(12)}$, including the following questions: (1) Do you have difficulty in seeing during the day? (2) Do you have difficulty in seeing in decreased light or at night? (3) Do you have NB? 
Question 3 was explained to the interviewees as an alteration in their habitual sight pattern in decreased light or at night by adopting the patient's pre-CLD nocturnal vision as the reference. Cases were defined as subjects who answered 'no' to question 1 and 'yes' to questions 2 and/or 3 because this ocular symptom reflects the functional role of vitamin $\mathrm{A}$ in the formation of rhodopsin.

\section{Assessment of biochemical parameters of liver function and liver damage}

The laboratory data collected included albumin, total bilirubin, aspartate transaminase (AST) and alanine transaminase (ALT) and prothrombin time, which were measured by previously established standard laboratory methods. The AST:ALT ratio was then calculated for each patient.

\section{Assessment of liver complications and death}

Clinical variables recorded were degree of ascites and hepatic encephalopathy based on ultrasound data and clinical evaluation, respectively. Laboratory data and degree of ascites and hepatic encephalopathy were used to calculate the ChildPugh score. The patients were then stratified as A, B or C in accordance with the Child-Pugh classification criteria ${ }^{(16)}$.

\section{Anthropometric measurements}

Nutritional status was evaluated based on mid-arm muscle circumference (MAMC) and triceps skinfold thickness (TST) to assess muscle protein and adipose tissue, respectively. Midarm circumference and TST were measured with a measuring tape and a Lange skinfold caliper, respectively. To minimise practical variability, the average of three consecutive measurements for TST was recorded. MAMC was calculated according to formulas described by Frisancho based on mid-arm circumference and TST measurements ${ }^{(17)}$. Malnutrition was defined as MAMC and/or TST below the 10th percentile ${ }^{(18)}$

\section{Statistical analysis}

The distribution of the referred values was identified as not normal. Statistical comparisons for significance were made using the Kruskal-Wallis non-parametric test as appropriate. The Mann-Whitney test was used to compare the numeric variables between the two groups. Associations between the categorical variables were performed by $\chi^{2}$ test. A receiveroperating characteristic analysis was used to determine the serum retinol threshold with the best death predictive value. Significance level was set at $P<0 \cdot 05$. Analyses were performed with SPSS 13.0 (SPSS, Inc., Chicago, IL, USA).

\section{Results}

\section{Serum retinol levels and night blindness frequency}

A total of 140 patients with HCV infection were enrolled and sixty-nine (49.3\%) of them were male. Median age was
55 years, varying from 33 to 76 years, with $30 \cdot 6 \%$ of the sample in the 31-to-50-year-old range, $66.6 \%$ in the 51-to70 -year-old range and $2 \cdot 8 \%$ over 70 years of age. There was no relationship between age and serum retinol concentration $(P=0 \cdot 100)$. Data analysis revealed that serum retinol concentrations did not differ according to $\operatorname{sex}(P=0 \cdot 060)$. The frequency of $\mathrm{VAD}$, low vitamin A intake, NB and undernourishment is shown in Table 1 . In the present study, VAD frequency was $54.3 \%$ of all the patients. For more than half $(54.0 \%)$ of the patients, vitamin A intake was below the RDA.

Median serum retinol concentration among the participants was $1.71 \mu \mathrm{mol} / \mathrm{l}$ (range $0 \cdot 180-3.626$ ) among the chronic hepatitis C patients, $1.00 \mu \mathrm{mol} / \mathrm{l}$ (range 0.62-2.50) for Cirrhosis Child-Pugh A patients, $0.97 \mu \mathrm{mol} / 1$ (range $0 \cdot 17-2 \cdot 11$ ) for Cirrhosis Child-Pugh B patients, $0.63 \mu \mathrm{mol} / 1$ (range 0.06-1.97) for Cirrhosis Child-Pugh $C$ patients and 0.56 (range $0 \cdot 10-$ 1.33) for Cirrhosis HCC patients. The median retinol levels observed in each stage of liver disease are presented in Fig. 1. There was a progressive and statistically significant drop in serum retinol levels according to liver disease severity $(P<0 \cdot 010)$.

In the present study, a greater prevalence of severe VAD in Cirrhosis Child-Pugh C and HCC patients was observed, demonstrating that the lowest vitamin A values are found during more advanced stages of the disease. Only in the hepatitis group was there an elevated percentage of individuals with adequate levels of the vitamin. Among the other groups, at most $50 \%$ of the individuals were observed to have adequate serum retinol levels (Table 2).

\section{Biochemical and anthropometric parameters}

In comparing median biochemical and anthropometric variables between the groups with adequate and inadequate serum retinol levels, the individuals with inadequate retinol presented a significantly higher median AST, AST:ALT ratio, total bilirubin and prothrombin time $(P=0 \cdot 030,0 \cdot 020,0 \cdot 010$ and 0.020 , respectively), with no significant difference for albumin levels, ALT levels, MAMC and TST values $(P=0.060$, 0.570, 0.590 and 0.092, respectively; Table 3).

Table 1. Frequency of vitamin $A$ deficiency (VAD), low vitamin $A$ intake, night blindness (NB) and undernutrition among patients with hepatitis $C$ virus-related chronic liver disease

(Number of patients and percentages)

\begin{tabular}{lrrr}
\hline Variables & Total & $n$ & $\%$ \\
\hline VAD $^{*}$ & & & \\
Chronic hepatitis & 29 & 8 & $27 \cdot 6$ \\
Cirrhosis Child-Pugh A & 36 & 19 & $52 \cdot 8$ \\
Cirrhosis Child-Pugh B & 30 & 15 & $50 \cdot 0$ \\
Cirrhosis Child-Pugh C & 30 & 21 & $70 \cdot 0$ \\
HCC & 15 & 13 & $86 \cdot 7$ \\
Vitamin A intake <RDA & 140 & 76 & $54 \cdot 0$ \\
NB & 140 & 26 & $18 \cdot 5$ \\
Undernutrition & 140 & 29 & $19 \cdot 2$ \\
\hline
\end{tabular}

HCC, hepatocellular carcinoma.

* Serum retinol $<1.05 \mu \mathrm{mol} / \mathrm{l}$. 


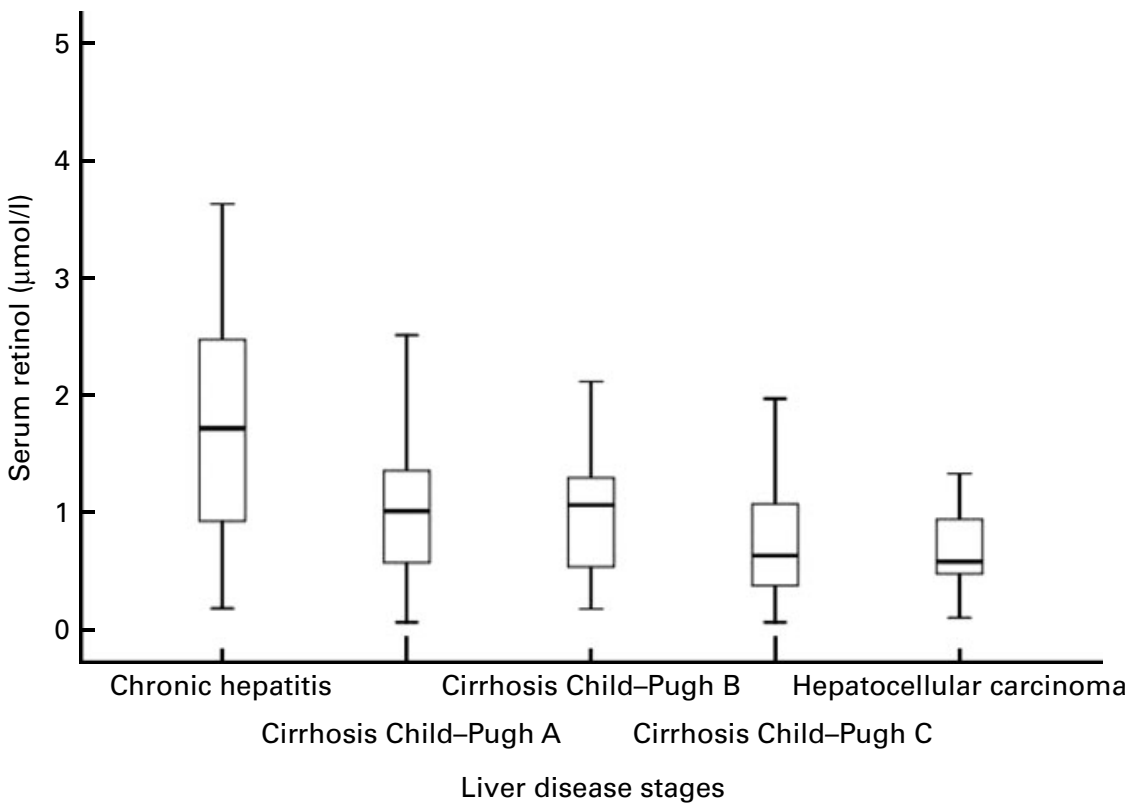

Fig. 1. Median of serum retinol levels observed in each liver disease stage. Mean values were significantly decreasing from chronic hepatitis $C$ patients towards patients with cirrhosis and hepatocellular carcinoma (Kruskal-Wallis; $P<0.010$ ).

\section{Liver complications and death}

Median serum retinol was significantly lower in patients with hepatic encephalopathy and ascites $(P=0.040$ and 0.006, respectively). There were forty-six patients who died during the period of study. Furthermore, those who died presented the lowest statistically significant value of serum retinol $(P=0.001 ;$ Table 4). There was a significant association between vitamin A nutritional status and liver-related death $(P=0 \cdot 026$; Table 5).

In the present study, according to the receiver-operating characteristic curve, it was observed that the best cut-off point for classifying death $v$. non-death serum retinol levels was $\leq 0.78 \mu \mathrm{mol} / 1$, with $70.7 \%$ sensibility and $52.8 \%$ specificity. The area under the receiver-operating characteristic curve was $71 \%$ (Fig. 2).

OR analysis shows that individuals below the $0.78 \mu \mathrm{mol} / 1$ cut-off point present 1.86 times greater likelihood of dying when compared with individuals over this cut-off point.

\section{Dietary vitamin A intake}

The distribution of dietary vitamin A intake during each stage of liver disease is shown in Table 6. An elevated proportion of low intake was observed in all stages of CLD, especially in the chronic hepatitis group. Taking all of the patients into consideration, about $70 \%$ of vitamin A intake came from vegetables and fruits and only $30 \%$ came from animal sources.

\section{Discussion}

The present study found a significant prevalence of VAD in the CLD of HCV patients, with intermediate values of those previously reported $^{(9,19)}$. This is the first study that endeavours to investigate the nutritional status of vitamin A according to biochemical, functional and dietetic indicators in chronic hepatitis, Child-Pugh A, B and C cirrhosis and HCC in HCV patients.

In the present study, neither age nor sex was linked to serum retinol level, an observation consistent with a past study $^{(20)}$. VAD prevalence was $54.3 \%$, which is consistent with the findings of Paula et $a l .^{(21)}$, who evaluated the nutritional status of vitamin A in fifty-eight patients with hepatic cirrhosis and observed low serum levels in $60 \%$ of the sample group, pointing out that this effort did not cover the initial stage of CLD, i.e. chronic hepatitis.

According to biochemical indicators, the present results confirm the progressive decrease in serum retinol with the

Table 2. Distribution of vitamin A deficiency considering all stages of hepatitis C virus-related chronic liver disease

\begin{tabular}{lcccc}
\hline Liver disease stages & $\geq 1.05 \mu \mathrm{mol} / \mathrm{l}$ & $<1.05-0.70 \mu \mathrm{mol} / \mathrm{l}$ & $0.69-0.35 \mu \mathrm{mol} / \mathrm{l}$ & $<0.35 \mu \mathrm{mol} / \mathrm{l}$ \\
\hline Chronic hepatitis (\%) & 72.40 & 17.20 & 5.60 & 3.40 \\
Cirrhosis Child-Pugh A (\%) & 47.20 & 25.00 & 10.00 & 5.60 \\
Cirrhosis Child-Pugh B (\%) & 50.00 & 10.00 & 30.00 & 10.00 \\
Cirrhosis Child-Pugh C (\%) & 30.00 & 16.70 & 33.30 & 20.00 \\
HCC (\%) & 13.30 & 20.00 & 46.70 & 20.00 \\
\hline
\end{tabular}

HCC, hepatocellular carcinoma. 
Table 3. Comparison of medians of the biochemical and anthropometric parameters between the groups with inadequate and adequate serum retinol levels

(Medians, minimum and maximum values)

\begin{tabular}{|c|c|c|c|c|c|}
\hline \multirow[b]{2}{*}{ Variables } & \multicolumn{2}{|c|}{$\begin{array}{l}\text { Inadequate serum retinol } \\
<1.05 \mu \mathrm{mol} / /\end{array}$} & \multicolumn{2}{|c|}{$\begin{array}{l}\text { Adequate serum retinol } \\
\quad \geq 1.05 \mu \mathrm{mol} / \mathrm{l}\end{array}$} & \multirow[b]{2}{*}{$P^{*}$} \\
\hline & Median & Minimum-maximum & Median & Minimum-maximum & \\
\hline MAMC $(\mathrm{cm})$ & $28 \cdot 17$ & $10 \cdot 31-84 \cdot 00$ & 29.83 & $9 \cdot 42-72 \cdot 20$ & 0.590 \\
\hline TST $(\mathrm{mm})$ & $15 \cdot 00$ & $5-30$ & 19.00 & $4 \cdot 0-32 \cdot 0$ & 0.092 \\
\hline AST $(U / I)$ & 93.00 & $25-211$ & 68.50 & $17-368$ & 0.030 \\
\hline $\operatorname{ALT}(\mathrm{U} / \mathrm{I})$ & $80 \cdot 00$ & $11-278$ & 73.00 & $14-233$ & 0.570 \\
\hline AST:ALT ratio & 1.01 & $0.09-3.11$ & 0.87 & $0.12-4.78$ & 0.020 \\
\hline Albumin (g/l) & $33 \cdot 0$ & $10-45$ & $35 \cdot 5$ & $19 \cdot 0-45 \cdot 0$ & $00 \cdot 60$ \\
\hline Total bilirubin (mg/l) & 14.5 & $03 \cdot 0-60.0$ & $10 \cdot 0$ & $04 \cdot 0-81 \cdot 0$ & $00 \cdot 10$ \\
\hline PTT (seconds above control) & 3.10 & $0.00-10.00$ & $2 \cdot 10$ & $0.0-7.90$ & 0.020 \\
\hline
\end{tabular}

MAMC, mid-arm muscle circumference; TST, triceps skinfold thickness; AST, aspartate transaminases; ALT, alanine transaminases;

PTT, prothrombin time.

* Mean values significantly different between the groups were calculated using the Mann-Whitney $U$ test $(P<0.050)$.

increase in CLD severity, and this finding corroborates prior studies involving CLD patients ${ }^{(20,22)}$. Serum retinol levels were significantly higher in patients diagnosed with chronic hepatitis C. It is worth noting that only the chronic hepatitis $C$ group bore median serum retinol values higher than the $1.05 \mu \mathrm{mol} / 1$ cut-off point. Also prominent is how VAD progresses according to the stage of the illness, and that the Child-Pugh C and HCC groups present a greater prevalence of severe $\mathrm{VAD}$, corroborating the findings of Paula et al. ${ }^{(21)}$ who reported low levels of retinol in $67 \%$ of the patients with advanced hepatic cirrhosis.

This progressive drop in serum vitamin A levels found in the present study could be a consequence of reduced amounts of vitamin A in the hepatic stellate cells due to activation by the HCV. This activation is a transition of quiescent cells into proliferative, fibrogenic and contractile myofibroblasts, although, at the moment, no definitive studies establish a causal link between loss of retinoid stores and progression of hepatic disease $^{(23,24)}$. Other factors associated with low serum retinol concentrations in CLD, such as poor dietary vitamin A intake and malabsorption of fat-soluble vitamins, can be speculated on; however, cholestasis was an exclusion criterion in the present study. It is further suggested that in CLD, there is a reduction in hepatic synthesis of the retinol carrier protein due to dysfunction of the organ or protein-energy malnutrition $^{(9,25)}$.
It is also possible that the demand for the vitamin increases with the progression of CLD. The serum and livers of individuals with HCV present an increase in lipid peroxidation products, such as malondialdehyde and hydroxynoneal ${ }^{(26)}$, and it has been suggested that there is an association between the production of these products and the activation of stellate cells. Thus, the depletion of antioxidants, such as vitamin A, may be related to greater need due to the oxidative process, bringing about an imbalance in the cellular redox balance ${ }^{(27)}$. In cirrhosis or chronic hepatitis, hepatocytes are in intense regenerative activity, and the loss of vitamin A, which contributes to the regulation and maintenance of hepatocyte differentiation, may result in the formation of mutant hepatocytes that may be progenitors to HCC cells.

Indeed, in studies carried out by Newsome et al. ${ }^{(20)}$ and Clemente et $a l .{ }^{(22)}$, it was noted that cirrhotic patients with HCC presented significantly lower levels of retinol than cirrhotic patients without HCC, suggesting that retinol deficiency may predispose HCC development in high-risk patients; for example, those with cirrhosis. Hence, serum retinol levels have been suggested as indicators of individuals at greater risk of developing HCC.

Traditional biochemical variables for assessing liver function $^{(28)}$, such as total bilirubin and prothrombin time, were significantly higher in individuals with inadequate serum retinol levels. There was no statistically significant

Table 4. Comparison of serum retinol levels between the groups with the presence or absence of hepatic encephalopathy, ascites and liver-related death

(Medians, minimum and maximum values)

\begin{tabular}{|c|c|c|c|c|c|}
\hline \multirow[b]{2}{*}{ Variables } & \multicolumn{2}{|r|}{ Presence } & \multicolumn{2}{|r|}{ Absence } & \multirow[b]{2}{*}{$P^{*}$} \\
\hline & Median & Minimum-maximum & Median & Minimum-maximum & \\
\hline \multicolumn{6}{|l|}{ Hepatic encephalopathy } \\
\hline Serum retinol $(\mu \mathrm{mol} / \mathrm{l})$ & 0.77 & $0.08-1.97$ & 1.01 & $0.06-3.62$ & 0.040 \\
\hline \multicolumn{6}{|l|}{ Ascites } \\
\hline Serum retinol $(\mu \mathrm{mol} / \mathrm{l})$ & 0.76 & $0.08-2.39$ & $1 \cdot 11$ & $0.06-3.62$ & 0.006 \\
\hline \multicolumn{6}{|l|}{ Death } \\
\hline \multicolumn{6}{|l|}{ Serum retinol $(\mu \mathrm{mol} / \mathrm{l})$} \\
\hline Yes & 0.64 & $0.08-1.97$ & & & 0.001 \\
\hline No & & & 1.07 & $0.06-3.62$ & \\
\hline
\end{tabular}

* Mean values significantly different between groups were calculated using the Mann-Whitney $U$ test $(P<0 \cdot 050)$. 
Table 5. Association between serum retinol levels and liver-related death

\begin{tabular}{lccccccc}
\hline & \multicolumn{2}{c}{ Vitamin A nutritional status } & & & & \\
\cline { 2 - 5 } Death & $<1.05 \mu \mathrm{mol} / /$ & $<1.05 \mu \mathrm{mol} / /$ & & & & \\
\hline No & $n$ & $n$ & Total & OR & $\chi^{2}$ & $P^{*}$ \\
Yes & 41 & 46 & 87 & 1.86 & 4.97 & 0.026 \\
Total & 31 & 15 & 46 & & & \\
& 72 & 61 & 133 & & & \\
\hline
\end{tabular}

* Mean values significantly different between groups were calculated using the Mann-Whitney $U$ test $(P<0.050)$.

difference in albumin levels, although there is a tendency towards reduced albumin levels in patients with inadequate serum retinol levels. Rocchi et $a l^{(29)}$ and Chaves et $a l^{(30)}$ found a significant negative correlation between serum retinol and total bilirubin in individuals with cirrhosis and nonalcoholic fatty liver disease, respectively. In the present study, in individuals with inadequate serum retinol levels, liver damage tests such as AST and AST:ALT ratio presented a significantly higher median, whereas ALT median values showed no difference. Previous studies have shown that an AST:ALT ratio $>1$ suggested cirrhosis $^{(31,32)}$. It has been suggested that with progressive fibrosis and cirrhosis, sinusoidal function is progressively hampered, reducing the clearance of AST from the serum ${ }^{(33)}$. In fact, Sheth et al. ${ }^{(34)}$ observed that the AST:ALT ratio correlated positively with the stage of fibrosis. So it could be postulated that retinol is a potential marker of liver damage and liver function, although the present data have not produced enough evidence to establish that conclusively.

The present study encountered a significant drop in serum retinol concentration among individuals suffering ascites and hepatic encephalopathy. It is worth stressing that these complications are common in advanced liver disease and relate to the largest number of hospital visits and the greatest incidence of death. The incidence of death is also associated with the presence of VAD. Such evidence substantiates increased risk of mortality among risk populations with VAD and without ocular signs, suggesting the need to reassess the cut-off points and criteria for interpreting serum retinol concentration distribution. In the present study, the cut-off point best associated with the risk of death was $0.78 \mu \mathrm{mol} / \mathrm{l}$, which is above the value considered to be moderate VAD by the World Health Organization ${ }^{(12)}$, reinforcing the need to reassess cut-off points for CLD patients.

The present findings show that dietary vitamin A intake is notably insufficient, with intake below the RDA in $54 \%$ of the sample group. Average intake was insufficient both in the chronic hepatitis and in the cirrhosis Child-Pugh $\mathrm{C}$ patients. Although only the chronic hepatitis $\mathrm{C}$ group presented physiologically acceptable circulating retinol levels, this reduced dietary vitamin A intake contributes to the depletion of retinoid storage in the early stage of CLD. It could be suggested that this insufficient vitamin A intake may contribute to the progression of liver disease, as oxidative stress is an important pathophysiological mechanism in patients infected by $\mathrm{HCV}$ and vitamin $\mathrm{A}$ is recognised to have important antioxidant $\operatorname{action}^{(35)}$. Rocchi et al. ${ }^{(23)}$ and Ukleja et $a l .{ }^{(19)}$ noted low levels of antioxidants in HCC patients when compared with control groups. Furthermore, vitamin A controls hepatocellular proliferation and differentiation ${ }^{(36)}$. Thus, considering the importance of the vitamin in the disease progression process and in chemoprevention of hepatocarcinogenesis ${ }^{(37)}$, it is important to guarantee adequate intake of the vitamin during the early stages of the disease.

It is worth pointing out that there was a high prevalence of inadequate intake in the Child-Pugh A, B and HCC groups, which could justify the reduction in serum retinol observed, although intake medians are within the recommended values, suggesting that consumption within what is recommended may not be enough to fight against oxidative stress.

Food frequency data revealed that a large proportion of vitamin A intake came from vegetables and fruits, while only $30 \%$ came from animal sources. Retinol is derived primarily from animal-based food, whereas vegetables and fresh fruit are major sources of provitamin A carotenoids. Provitamin A carotenoids can be converted to retinol in vivo. The mean conversion rate is of approximately $12 \mu \mathrm{g}$ of $\beta$-carotene and $24 \mu \mathrm{g}$ of other carotenoids to $1 \mu \mathrm{g}$ of retinol in healthy adults. The conversion of $\beta$-carotene to retinol, for it occurring in the liver, may be deficient in these patients, contributing to the lowered levels of serum retinol in this group ${ }^{(12)}$.

Moreover, this low intake of animal sources also contributes to the reduced serum retinol levels observed in CLD patients. Dietary beliefs, taboos and constraints associated with liver disease lead to reduced intake of protein and fat and, consequently, compromise the intake and absorption of preformed vitamin A, as well as other micronutrients ${ }^{(38)}$.

By applying the questionnaire for functionally evaluating the nutritional status of vitamin $\mathrm{A}$, an $18.5 \%$ prevalence of NB was detected in the sample group. NB is the first functional manifestation of VAD that can be potentially measured before the drop in serum levels ${ }^{(12,39)}$. Patek \& Haig ${ }^{(40)}$, using an

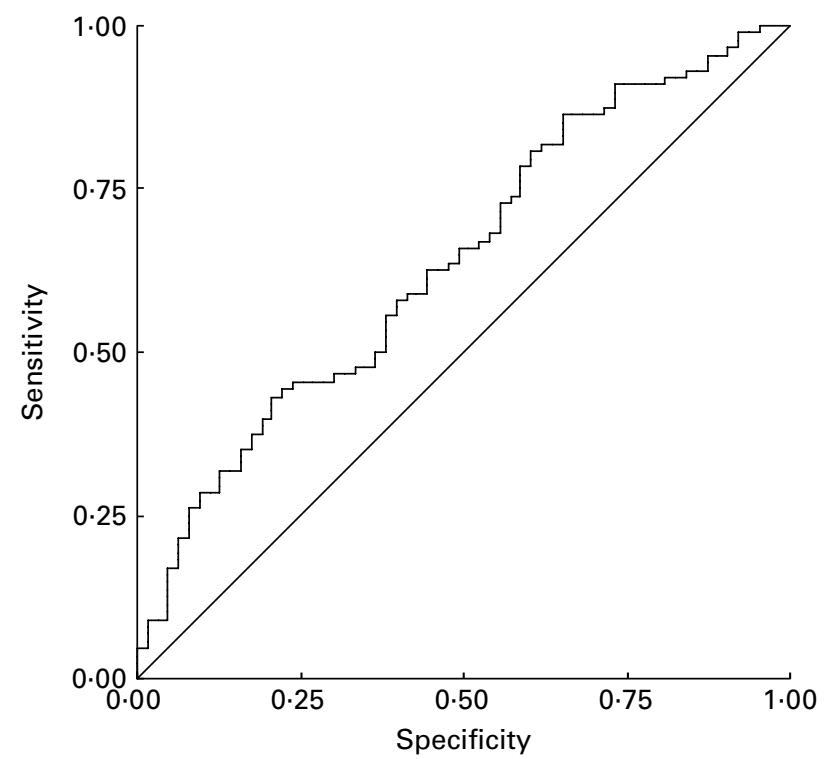

Fig. 2. Receiver-operating characteristic curve of retinol as predictor of death. Area under the curve, 0.71. 
Table 6. Vitamin A intake among patients with hepatitis $C$ virus-related chronic liver disease

(Medians and ranges)

\begin{tabular}{lccc}
\hline & \multicolumn{3}{c}{ Dietary vitamin A intake $(\mu \mathrm{g} / \mathrm{d})^{\star}$} \\
\cline { 2 - 4 } Study groups & Median & Range & $\begin{array}{c}\text { Low ingestion } \\
\text { of vitamin A (\%) }\end{array}$ \\
\hline Chronic hepatitis & 561.82 & $115.12-2025.47$ & 63.6 \\
Cirrhosis Child-Pugh A & 993.32 & $235.90-227.80$ & 40.7 \\
Cirrhosis Child-Pugh B & 894.88 & $245 \cdot 60-2415.60$ & 40.0 \\
Cirrhosis Child-Pugh C & 685.61 & $144.70-1809.72$ & 59.1 \\
HCC & 872.40 & $233.86-1323.72$ & 37.5 \\
\hline
\end{tabular}

HCC, hepatocellular carcinoma.

${ }^{*}$ RDA: $900 \mathrm{mg} / \mathrm{d}$ for males and $700 \mathrm{mg} / \mathrm{d}$ for females.

adaptometer for measuring human dark adaptation, described an elevated prevalence of $\mathrm{NB}$ in cirrhosis patients (79.2\%), and Ukleja $e t a l .{ }^{(19)}$, through the application of a pre-validated questionnaire, found a prevalence of $22 \%$. More recently, Mahmood et al. ${ }^{(41)}$ have reported NB in $47 \%$ of individuals with cirrhosis, also describing cases of conjunctival xerosis and Bitot's spots in some of the individuals studied. In the present study, one possible explanation for the low prevalence of NB may be the high prevalence of sight problems, which may lead to misclassification of NB. Furthermore, NB diagnosis in the present study was performed by applying a validated questionnaire for classic risk groups, which may not be the most appropriate method for CLD patients.

In summary, a biochemical indicator was demonstrated to be an important parameter for evaluating vitamin A nutritional status in CLD patients, for its association with the severity of the disease, liver complications and mortality, beyond which a high prevalence of insufficient vitamin A intake was observed, especially in the initial stage of CLD, i.e. chronic hepatitis. This finding highlights the need for measures of intervention in this group so as to improve the nutritional status of this population, which would result in greater oxidative protection and lower risk of complications from the disease and the development of HCC, but further studies are necessary to confirm it or dismiss it. In the present study, the functional indicator was not a good parameter for assessing the vitamin A nutritional status, suggesting a need to evaluate the pertinence of applying the questionnaire proposed by the WHO for diagnosing NB in CLD patients.

\section{Acknowledgements}

The present study was supported by the Brazilian research support agencies, Fundação Carlos Chaga Filho de Amparo à Pesquisa do Estado do Rio de Janeiro (FAPERJ) and Conselho Nacional de Desenvolvimento Científico e Tecnológico (CNPq). W. A. F. P., A. R. and H. S. M. C. conceived of and coordinated the study. W. A. F. P. and J. C. S. G. carried out the biochemical, functional, dietetics and statistical analyses. W. A. F. P. and G. V. C. recruited the participants and collected the data. All authors contributed to the writing and reviewing of the manuscript and approved the final version. There are no conflicts of interest.

\section{References}

1. Bhopale GM \& Nanda RK (2005) Emerging drugs for chronic hepatitis C. Hepatol Res 32, 146-153.

2. Consensus statement (1999) EASL International consensus conference on hepatitis C. J Hepatol 31, Suppl. 1, S3-S8.

3. Leigh JP, Bowlus CL, Leistikow BN, et al. (2001) Costs of hepatitis C. Arch Intern Med 161, 2231-2237.

4. Poynard T, Munteanu M, Imbert-Bismut F, et al. (2004) Prospective analysis of discordant results between biochemical markers and biopsy in patients with chronic hepatitis C. Clin Chem 50, 1344-1355.

5. World Health Organization (1995) Global prevalence of vitamin A deficiency. Micronutrient Deficiencies Information System - Working Paper No. 2. Document WHO/NUT/95.3. Geneva: WHO.

6. Friedman SL (2000) Molecular regulation of hepatic fibrosis, an integrated cellular response to tissue injury. $\mathrm{J} \mathrm{Biol} \mathrm{Chem}$ 28, 2247-2250.

7. Milliano MT \& Luxon BA (2005) Rat hepatic stellate cells become retinoid unresponsive during activation. Hepatol Res 33, 225-233.

8. International Vitamin A Consultative Group (2003) IVACG statement. Improving the Vitamin A Status of Populations. Washington, DC: International Vitamin A Consultative Group.

9. Russell RM, Iber FL, Krasinski SD, et al. (1983) Proteinenergy malnutrition and liver dysfunction limit the usefulness of the relative dose response (RDR) test for predicting vitamin A deficiency. Hum Nutr Clin Nutr 37, 361-371.

10. Bataller R \& Brenner DA (2005) Liver fibrosis. J Clin Invest 115, 209-218.

11. Pinzani M \& Rombouts K (2004) Liver fibrosis from bench to clinical targets. Dig Liver Dis 36, 231-242.

12. World Health Organization (1996) Global prevalence of vitamin A. Indicators for assessing vitamin $A$ deficiency and their application in monitoring and evaluating intervention programmes. Micronutrient Series, WHO/NUT1O. Geneva: WHO.

13. International Vitamin A Consultative Group (2003) Improving the vitamin A status of populations. USAID-ILSI

14. Coelho CS, Ramalho RA \& Accioly E (1995) Vitamina A: inquérito dietético na avaliacão do estado nutricional em gestantes (Vitamin A: dietary survey in the evaluation of nutritional status in pregnant women). Gynaecia $\mathbf{1}$, 200-204.

15. Institute of Medicine (2001) Food and Nutrition Board. Vitamin A. In Dietary Reference Intakes for Vitamin A, Vitamin K, Arsenic, Boron, Chromium, Copper, Iodine, Iron, Vanadium, Manganese, Molybdenum, Nickel, Silicon, Vanadium and Zinc, pp. 82-161. Washington, DC: National Academy Press.

16. Pugh RN, Murray-Lyon IM, Dawson JL, et al. (1973) Transection of the oesophagus for bleeding oesophageal varices. Br J Surg 60, 646-649.

17. Frisancho AR (1981) New norms of upper limb fat and muscle areas for assessment of nutritional status. Am J Clin Nutr 34, 2540-2545.

18. Alberino F \& Gatta A (2001) Nutrition and survival in patients with liver disease. Nutrition 17, 445-450.

19. Ukleja A, Scolapio JS, McConnell JP, et al. (2002) Nutritional assessment of serum and hepatic vitamin A levels in patients with cirrhosis. JPEN 26, 184-188.

20. Newsome PN, Beldon I, Moussa Y, et al. (2000) Low serum retinol levels are associated with hepatocellular carcinoma in 
patients with chronic liver disease. Aliment Pharmacol Ther 14, 1295-1301.

21. Paula TP, Ramalho A \& Braulio VB (2010) The effectiveness of relative dose response to retinol intake as an evaluation of vitamin A status of cirrhotic patients. J Hum Nutr Diet 6, 1-7.

22. Clemente C, Elba S, Buongiorno G, et al. (2002) Serum retinol and risk of hepatocellular carcinoma in patients with Child-Pugh class A cirrhosis. Cancer Lett 178, 123-129.

23. Rocchi E, Casalgrandi G, Ronzoni A, et al. (2001) Antioxidant liposoluble vitamins and carotenoids in chronic hepatitis. Eur J Intern Med 12, 116-121.

24. Yadav D, Hertan HI, Schweitzer P, et al. (2002) Serum and liver micronutrient antioxidants and serum oxidative stress in patients with chronic hepatitis C. Am J Gastroenterol 97, 2634-2639.

25. Calamita Z, Dichi I, Papini-Berto SJ, et al. (1997) Plasma levels of transthyretin and retinol-binding protein in childA cirrhotic patients in relation to protein-calorie status and plasma amino acids, zinc, vitamin A and plasma thyroid hormones. Arq Gastroenterol 34, 139-147.

26. De Maria N, Calantoni A, Fagiuoli S, et al. (1996) Association between reactive oxygen species and disease activity in chronic hepatitis C. Free Radic Biol Med 21, 291-295.

27. Loguercio C \& Federico A (2003) Oxidative stress in viral and alcoholic hepatitis. Free Radic Biol Med 34, 1-10.

28. Mecocci P, Polidori MC, Troiano L, et al. (2000) Plasma antioxidants and longevity: a study on healthy centenarians. Free Radic Biol Med 28, 1243-1248.

29. Rocchi E, Borghi A, Paolillo F, et al. (1991) Carotenoids and lipossoluble vitamins in liver cirrhosis. J Lab Clin Med $\mathbf{1 1 8}$, $176-185$.

30. Chaves GV, Pereira SE, Saboya CJ, et al. (2008) Nonalcoholic fatty liver disease and its relationship with the nutritional status of vitamin $\mathrm{A}$ in individuals with class III obesity. Obes Surg 18, 378-385.
31. Cohen JA \& Kaplan MM (1979) The SGOT/SGPT ratio - an indicator of alcoholic liver disease. Dig Dis Sci 24, 835-838.

32. Williams AL \& Hoofnagle JH (1988) Ratio of serum aspartate to alanine aminotransferase in chronic hepatitis. Relationship to cirrhosis. Gastroenterology 95, 734-739.

33. Kamimoto Y, Horiuchi S, Tanase S, et al. (1985) Plasma clearance of intravenously injected aspartate aminotransferase isozymes: evidence for preferential uptake by sinusoidal liver cells. Hepatology 5, 367-375.

34. Sheth SG, Flamm SL, Gordon FD, et al. (1998) AST/ALT ratio predicts cirrhosis in patients with chronic hepatitis $\mathrm{C}$ virus infection. Am J Gastroenterol 93, 44-48.

35. Fierbinteanu-Braticevici C, Mohora M, Cretoiu D, et al. (2009) Role of oxidative stress in the pathogenesis of chronic hepatitis C (CHC). Rom J Morphol Embryol 50, 407-412.

36. Gudas LJ (1992) Retinoids, retinoid-responsive genes, cell diferentiation and cancer. Cell Growth Dif 3, 655-662.

37. Mangelsdorf DJ (1994) Vitamin A receptors. Nutr Rev 52 $32-44$.

38. Cincinatus R, Chaves GV, Aquino LA, et al. (2007) Consumo dietético de macronutrientes e micronutrientes e a sua relação com a gravidade da doença hepática. Rev Soc Bras Alim Nutr 32, 61-77.

39. Mclaren DS \& Frigg M (1999) Manual de ver y vivir sobre los transtornos por deficiencia de vitamina A (VADD) (Manual of Seeing and Living on the Disorders due to Vitamin $A$ (VADDER)). Washington, DC: Organización Panamericana de la Salud.

40. Patek AJ \& Haig C (1939) The occurrence of abnormal dark adaptation and its relation to vitamin A metabolism in patients with cirrhosis of the liver. J Clin Invest 18, 609-619.

41. Mahmood K, Samo AH, Jairamani KL, et al. (2008) Serum retinol binding protein as an indicator of vitamin A status in cirrhotic patients with night blindness. Saudi J Gastroenterol 14, 7-11. 